\title{
Familien und Organisationen: Gemeinsamkeiten, Unterschiede und Verknüpfungen
}

\author{
Stefan Kühl
}

\section{Einleitung - Jenseits des Schemas von Interaktion, Organisation und Gesellschaft}

Die Stärke der Systemtheorie ist, dass sie die unterschiedlichen Logiken in verschiedenartigen sozialen Systemen begreifbar machen und für ihre Analysen nutzen kann. In der Systemtheorie werden bei der Behandlung moderner Gesellschaften immer nur zwei Differenzierungsformen behandelt - die funktionale Differenzierung zwischen unterschiedlichen gesellschaftlichen Feldern wie Wirtschaft, Politik, Recht, Wissenschaft oder Massenmedien und die Ebenendifferenzierung zwischen Interaktionen, Organisationen und Gesellschaft.

Übersehen wird jedoch dabei, dass auf der Ebene zwischen Interaktionen und Gesellschaft die Unterschiede, Gemeinsamkeiten, Übergänge und Überschneidungen von unterschiedlichen Systemtypen wie Gruppen, Bewegungen, Organisationen oder Familien systematisch untersucht werden können. Statt „Zweierlei Differenzierungen“ (Tyrell 2008) lassen sich meines Erachtens deswegen systemtheoretisch „dreierlei Differenzierungen“ identifizieren. Auf der Ebene zwischen Interaktionen und Gesellschaft haben sich - so die These - nicht nur Organisationen ausdifferenziert, sondern auch andere soziale Zusammenhänge wie Gruppen, Bewegungen oder Familien. Inwiefern solche sozialen Zusammenhänge - man könnte auch noch Gemeinden oder Schichten - den Charakter von sozialen Systemen annehmen, muss systemtheoretisch noch näher bestimmt werden.

\footnotetext{
S. Kühl $(\bowtie)$

Universität Bielefeld, Bielefeld, Deutschland

E-Mail: stefan.kuehl@uni-bielefeld.de

(C) Springer Fachmedien Wiesbaden GmbH, ein Teil von Springer Nature 2019

H. Kleve und T. Köllner (Hrsg.), Soziologie der Unternehmerfamilie,

https://doi.org/10.1007/978-3-658-22388-5_4
} 
Ziel dieses Beitrages ist es, vor dem Hintergrund dieser Überlegungen die Differenz von Familien und Organisationen näher ins Auge zu fassen. Dafür werden im folgenden zweiten Abschnitt die grundlegenden Unterschiede von Familien und Organisationen herausgearbeitet. Im dritten Abschnitt wird gezeigt, wie sich Familien und Organisationen überhaupt als Systemtypen mit eigenen Logiken ausgebildet haben. Der abschließende vierte Abschnitt stellt in aller Kürze die analytischen Möglichkeiten dar, die man bei der Betrachtung der Verschachtelung von Familien und Organisationen hat.

\section{Unterschiedliche Konzeptionen von Mitgliedschaften}

Es fällt auf, dass alltagssprachlich nicht nur bei Organisationen, sondern auch bei Familien (aber auch bei Gruppen oder Protestbewegungen) von Mitgliedern gesprochen wird. Selbstverständlich spricht die alltagssprachliche Verwendung eines Begriffs allein noch nicht dafür, ihn dann auch für die soziologische Bestimmung sozialer Systeme zu verwenden. Aber die Verwendung des Mitgliedschaftsbegriffs auf eine Vielzahl sozialer Phänomene kann als Indiz dafür gewertet werden, dass es eine Vielzahl von mitgliedschaftsbasierten Sozialsystemen gibt (Martens 1997; Mahlert 2011).

Nicht nur bei Organisationen, sondern auch bei Familien erfolgt eine Zurechnung von Kommunikation darüber, ob eine Person als Mitglied betrachtet wird oder nicht. Personen können eben nicht nur in Organisationen, sondern auch in Familien als „Identifikationspunkte der Kommunikation“, als „Adressen für Kommunikation“, als „Einheiten der Handlungszurechnung“ dienen (Luhmann 2005a, S 194). ${ }^{1}$ Wenn man jetzt den Blick dafür öffnet, dass nicht nur Organisationen, sondern auch Familien Personen als Mitglieder identifizieren und damit Kommunikationen zurechenbar machen, muss erklärt werden, weswegen es sich um verschiedene soziale Systeme mit unterschiedlichen Logiken handelt.

Im Folgenden soll gezeigt werden, dass zwar sowohl Organisationen als auch Familien Personen identifizieren, die als Mitglied dazugehören (oder eben nicht), dass aber die Mitgliedschaften dieser Personen zu den jeweiligen sozialen Systemen unterschiedlich gehandhabt werden. Daraus ergeben sich dann nicht nur - was

\footnotetext{
${ }^{1}$ Der Begriff der „Person“ ist dabei für meine Zwecke präziser als der zurzeit in der Theoriediskussion populäre Begriff der „Adresse“ (vgl. Fuchs 1997 und im Anschluss an ihn zum Beispiel Tacke 2000 oder Stichweh 2000). Adresse ist der deutlich weitere Begriff, weil im Prinzip nicht nur Personen, sondern auch Tiere, Organisationen, Staaten und Geister adressiert werden können.
} 
spontan einleuchtend ist - verschiedene Formen der Grenzziehung der jeweiligen Systemtypen, sondern auch unterschiedliche Formen von Kommunikation, über die sich die verschiedenen sozialen Systeme reproduzieren.

\section{Organisationen}

Mitgliedschaft - die Entscheidung über einen Personenkreis, dessen Entscheidungen als Entscheidungen der Organisation wahrgenommen werden wird von den meisten Organisationsforschern unterschiedlicher theoretischer Ausrichtung als ein Merkmal von Organisationen angesehen (siehe zum Beispiel Caplow 1964, S. 1 f.; Mayntz und Ziegler 1977, S. 34). In der Systemtheorie wird die Mitgliedschaft jedoch nicht nur als ein, sondern als das zentrale Bestimmungsmerkmal von Organisationen benutzt. Organisationen, so Luhmann, bilden sich immer dann aus, wenn der „Eintritt ins System“ und der „Austritt aus dem System“ als „entscheidbar vorausgesetzt wird“ (vgl. Luhmann 1975c, S. 99; siehe auch Luhmann 1975a, S. 12).

Die Organisation kann also darüber entscheiden, wer zu einem Unternehmen, einer Verwaltung, einer Partei oder einem Sportverein gehört und wer nicht. Und folgenreicher: Sie kann darüber bestimmen, wer ihr nicht mehr angehören soll, weil er oder sie den Regeln der Organisation nicht mehr folgt. Die Organisation schafft Grenzen, in denen sich die Mitglieder (und eben nur die Mitglieder) den Regeln der Organisation zu unterwerfen haben, und es hängt permanent die Drohung im Raum, dass das Mitglied die Organisation zu verlassen hat, wenn es die Regeln nicht befolgt (Luhmann 1964, S. 44 f.). In Organisationen sind Entscheidungen über den Ein- und Austritt von Personen - die Bestimmung von Mitgliedschaften - ein zentrales Instrument, um konformes Verhalten ihrer Mitglieder herzustellen.

Durch die Möglichkeit, Mitgliedschaft zu konditionieren - d. h., ein Verhalten für alle erwartbar zu machen, indem man andernfalls mit Aufkündigung der Mitgliedschaft droht -, können Organisationen Entscheidungskommunikation als eine systemspezifische Form der Kommunikation ausbilden (vgl. Luhmann 2002, S. 160). ${ }^{2}$ Das bedeutet nicht, dass in Organisationen jede Kommunikation in Form der Entscheidungskommunikation stattfindet. Es wird in Organisationen häufig

\footnotetext{
${ }^{2}$ Bei Luhmann (1973, S. 44) heißt es schon früh, dass formale Organisation ,,jene evolutionäre Errungenschaft" ist, die es den Entscheidungsprozessen ermöglicht, reflexiv zu werden.
} 
wild debattiert, kritisiert und imaginiert. Aber die Besonderheit ist, dass durch die Konditionierung der Mitgliedschaft jede Kommunikation in eine Entscheidungskommunikation überführt werden kann. Damit unterscheidet sich die Kommunikation in Organisationen von der in Bewegungen, Familien und Gruppen, in denen zwar auch entschieden wird, in denen Entscheidungskommunikationen sich aber eben nicht in der gleichen Form rekursiv verknüpfen können.

\section{Familien}

Es gibt offensichtlich zwei Möglichkeiten, Mitglied einer Familie zu werden, und beide Möglichkeiten müssen genutzt werden, damit eine Familie überhaupt zustande kommt: die Bildung einer Beziehung zwischen in der Regel gegengeschlechtlichen Partnern und die Angliederung von Kindern entweder durch Geburt oder Adoption. Mit der Geburt oder Adoption eines Kindes im Rahmen einer Beziehung ist eine neue Familie also „,vollständig auf den Weg gebracht“. „Alle notwendigen Positionen - Vater, Mutter und Kind - sind besetzt, auch wenn sich diese Familie um weitere Kinder (aber nicht mehr um weitere Eltern) vermehren mag“ (so Tyrell 1983b, S. 364 in einer frühen, teilweise noch durch ein normatives Bild der Familie geprägten Bestimmung).

Die von Hartmann Tyrell später propagierte Bestimmung von Familien über eine häufig ,riskante Kopplung von Partnerschaft und Elternschaft" scheint eine Weiterentwicklung der überharmonischen Darstellung Vater - Mutter - Kind zu sein. Die Logik der Partnerschaft ist häufig eine ganz andere Logik als die der Elternschaft. In der Familie werden diese beiden Logiken gekoppelt (Tyrell und Herlth 1994, S. 1 ff.; siehe dazu auch Tyrell 2008, S. 317). ${ }^{3}$ Diese Aufspaltung der Bestimmung von Familien in Partnerschaft einerseits und Elternschaft andererseits ermöglicht es auch, über den Aspekt der Mitgliedschaft den Blick auf Familien genauer zu bestimmen. Bei Elternschaften können Mitgliedschaften nicht einfach aufgekündigt werden. Kinder können nicht einfach aus der Familie ausgeschlossen werden, wenn sie sich nicht entsprechend den Ansprüchen der Eltern verhalten. Und auch die eigene Kündigung der Familienmitgliedschaft

\footnotetext{
${ }^{3}$ Dieses Modell geht mit seiner Betonung der häufig entgegengesetzten Logiken von Partnerschaft und Elternschaft auf Distanz zum Konzept der Kernfamilie mit ,ihrer starken Einheitssuggestion“. Mit diesem interessanten Gedanken richtet sich Tyrell gegen die in der Familiensoziologie weitverbreitete Vorstellung von Familien als einer Triade aus Mutter, Vater und Kind (Tyrell 2008, S. 317, bei dem sich auch die entsprechenden Literaturangaben finden).
} 
durch die Kinder selbst fällt schwer. Kinder sind sich dieser Unmöglichkeit des Ausschlusses bewusst und nutzen dies mit spektakulären Widerstandsaktionen gegen ihre Eltern besonders bei großen Familienfesten oder in den Warteschlangen vor den Kassen des Supermarktes aus. Motto: Was soll schon passieren, die können mich ja nicht einfach entlassen. Bei Partnerschaften ist die Auflösung einer Mitgliedschaft nicht nur vorstellbar, sondern - jedenfalls in der modernen Gesellschaft - die Regel. Auch wenn man auf den ersten Blick den Eindruck bekommen kann, dass eine Beziehung - ähnlich wie die Mitgliedschaft in einer Organisation - „kündbar“ ist (Tyrell 2008, S. 317), muss doch der besondere Charakter von Partnerschaften im Auge behalten werden. In einer Beziehung ist es nur schwerlich möglich, beim Partner Verhaltensweisen einzuklagen, indem man ihm bzw. ihr mit Trennung droht. Wenn ein Partner die Fortführung der Partnerschaft unter die Bedingung stellt, dass im Haushalt regelmäßig geputzt wird, zukünftig vorsichtiger Auto gefahren wird oder auf weitere Liebesabenteuer mit anderen Geschlechtspartnern verzichtet wird, hat man es bereits mit deutlichen Krisenerscheinungen zu tun.

Trotz (oder vielleicht besser: gerade wegen) dieser Fragilität der Mitgliedschaft ist heute in Familien Intimkommunikation nicht nur in einem im Vergleich zur vormodernen Gesellschaft überraschend hohen Maße erlaubt, sondern geradezu gefordert (ausführlich und aufschlussreich Gilgenmann 1994, S. 66; siehe auch Luhmann 2005b, S. 213). ${ }^{4}$ Intimkommunikation bedeutet nicht, dass die Kommunikation in Familien durch ein permanentes Liebesgesäusel geprägt ist. Dafür gäbe es empirisch wenig Plausibilität. Vielmehr besagt Intimkommunikation, dass ,alles, was eine Person betrifft,“ prinzipiell „,ür Kommunikation zugänglich ist". Geheimhaltung könne, so Luhmann, von Eltern, aber auch von Kindern praktiziert werden, ,aber sie hat keinen legitimen Status“. Man könne in der Familie „eine Kommunikation über sich selbst nicht ablehnen mit der Bemerkung: das geht Dich nichts an!“ (Luhmann 2005a, S. 193).

\footnotetext{
${ }^{4}$ Dass Elternschaft und Partnerschafft die gleiche Kommunikationsform zugewiesen wird mag überraschen. Schließlich wurde der Begriff der Intimkommunikation ursprünglich nur für die, durch das romantische Liebesideal geprägte, Partnerschaft verwendet (Luhmann 1982) und dann später auf Familien als Ganzes - also auch für die Elternschaft - ausgeweitet (Luhmann 2005a). Auch wenn die Semantik in Partnerschaft und Elternschaft auf den ersten Blick überraschend ähnlich ist (so beispielsweise die Aussage „Ich liebe dich“, die sowohl Kindern als auch Partnern gegenüber verwendet wird), müssen Ähnlichkeiten und Unterschiede in der Intimkommunikation in Partnerschaft und Elternschaft noch näher untersucht werden (siehe Tyrell und Herlth 1994, S. 6 ff. zur „bürgerlichen Einheitssemantik“, die sicherlich für die Ähnlichkeit in der Kommunikationsform verantwortlich ist).
} 
Intimkommunikation unterscheidet sich von persönlicher Kommunikation dadurch - der Gedanke ist in der Systemtheorie bisher noch nicht ausgearbeitet -, dass in der Intimkommunikation der Anspruch besteht, alle anderen Rollen thematisieren zu können, während in der persönlichen Kommunikation nur der Anspruch erhoben werden kann, einige andere Rollen zu thematisieren. In einer Interaktion im Freundeskreis kann die Frage nach vielleicht skurril wirkenden religiösen Praktiken zurückgewiesen werden, während die Nichtbeantwortung der Frage in einer Paarbeziehung erklärungsbedürftig wäre.

\section{Gemeinsamkeiten und Unterschiede}

Die Gemeinsamkeit von Organisationen und Familien besteht darin, dass permanent gescannt wird, ob eine Person, die einen Kommunikationsbeitrag leistet, als Mitglied des sozialen Systems behandelt wird oder nicht. Im Gegensatz zum sozialen System der Gesellschaft, das auf das Prinzip der kommunikativen Erreichbarkeit ausgerichtet ist, bezeichnet Mitgliedschaft eben gerade die Unterscheidung von Zugehörigkeit oder Nichtzugehörigkeit zu einem System. Das Lamentieren einer Person oder das Schreien eines Babys hat - je nachdem, ob die Person als Mitglied betrachtet wird oder nicht - für ein System eine entsprechend grundlegend andere Bedeutung. Die Unterscheidung zwischen Mitgliedern und Nichtmitgliedern funktioniert also als „Erkennungssignal“ nicht nur von Organisationen, sondern auch von Familien (Luhmann 1991, S. 202).

Die Unterschiede zwischen Organisationen und Familien basieren jetzt aber wie gezeigt darauf, dass Mitgliedschaft (und damit auch Nichtmitgliedschaft) unterschiedlich bestimmt werden. Vereinfacht lässt sich sagen, dass Personen in Organisationen durch Entscheidungen über Ein- und Austritt Mitglieder werden und in Familien qua Geburt und Tod. Während bei Organisationen also Mitgliedschaft durch eine Kombination aus Selbstselektion (des Mitglieds) und Fremdselektion (der Organisation) zustande kommt, bestehen bei Familien dagegen - jedenfalls Kinder und Eltern - keine Möglichkeit der Selbst- oder Fremdselektion, sondern neue Mitglieder werden durch die Geburt einer Familie „zugewiesen“. Es gibt sicherlich immer wieder Grenzfälle - Organisationen, in denen man beispielsweise über freie Mitarbeiterschaft „schleichend“ Mitglied wird oder Familien, in denen sich die Kinder von ihren Eltern ,scheiden“ lassen - aber diese Graubereiche sprechen nicht gegen die Unterscheidung von Organisationen und Familien.

Aufgrund der unterschiedlichen Formen der Handhabung von Mitgliedschaft tendieren Familien und Organisationen dazu, unterschiedliche Kommunikationsformen auszubilden - in Organisationen Entscheidungskommunikation und in 
Familien Intimkommunikation. Die Ausbildung von eigenen Kommunikationsformen bedeutet weder, dass im jeweiligen sozialen System ausschließlich in der jeweils spezifischen Form kommuniziert wird, noch, dass die Kommunikationsform exklusiv in dem jeweiligen sozialen System zu finden ist. Auch in Familien wird manchmal entschieden, auch Organisationen kann es Momente persönlicher Kommunikation geben. Aber nur im jeweiligen sozialen System dient - das ist ein alter Gedanke Luhmanns - die jeweilige Kommunikationsform zur Reproduktion des betreffenden sozialen Systems.

\section{Die Ausdifferenzierung von Gruppen, Organisationen, Bewegungen und Familien}

Ein zentraler Gedanke der Luhmann'schen Gesellschaftstheorie ist, dass es beim Wandel von einer segmentären über eine stratifizierte zu einer funktional differenzierten Gesellschaft zu einer ,zunehmenden Differenzierung“ der Ebenen von Interaktion, Organisation und Gesellschaft gekommen ist. In den segmentär differenzierten archaischen Gesellschaftsformationen seien, so Luhmann, Interaktion, Organisation und Gesellschaft ,nahezu identisch gewesen“. Eine archaische Stammesgesellschaft bestehe aus dem „Umkreis absehbarer, für den Einzelnen zugänglichen Interaktionen“. Sie stoße - „wie eine Organisation“ - „Personen, die sich nicht fügen, aus" und nehme, ,vor allem durch Heirat, Personen auf“. In der stratifizierten Gesellschaft der Hochkulturen werde der „Umfang der für den Einzelnen möglichen Interaktionen“ gesprengt. In den städtischen Zentren bildeten sich insbesondere Organisationen ,für religiöse, politische, militärische, kommerzielle Funktionen oder für einzelne Produktionsaufgaben“. Weil aber „,der Zugriff der Organisationen auf die Lebensführung im Alltag gering“ sei und umgekehrt „die Gesellschaft selbst als politische Organisation, als handlungsfähige Kooperation“ begriffen werde, könne noch nicht von einer vollständigen Differenzierung der Ebenen von Interaktion, Organisation und Gesellschaft gesprochen werden: Diese bildete sich erst mit der Entstehung einer funktional differenzierten Gesellschaft. Gesellschaft könne dann weder „als Summe alltäglicher Begegnungen“ noch als „einheitliches Organisationssystem“ verstanden werden (vgl. Luhmann 1975a, S. 13).

Wie muss dieser hier in enger Anlehnung an Luhmanns eigene Formulierungen geschilderte Teil seiner Gesellschaftstheorie modifiziert werden, wenn man zwischen Interaktion und Gesellschaft verschiedene Systeme wie Bewegungen, Gruppen, Organisationen und Familien verortet? 


\section{Profilierung von Familien und Organisationen als soziale Systeme gegeneinander}

Eine Rückprojektion von für die moderne Gesellschaft typischen sozialen Systemen auf segmentäre oder stratifizierte Gesellschaften ist problematisch. Man kann sicherlich darauf aufmerksam machen, dass segmentäre Gesellschaften und stratifizierte Gesellschaften (ähnlich wie moderne Organisationen) Personen, die sich nicht fügen wollen, ausstoßen, aber damit erschöpfen sich auch schon die analytischen Vergleichsmöglichkeiten. Insofern muss man vorsichtig damit sein, soziale Systeme, die für die moderne Gesellschaft typisch sind, wie Organisationen oder nur noch aus einem Elternpaar und Kindern bestehende Familien für die Analyse segmentärer oder stratifizierter Gesellschaften zu nutzen. Vielmehr ist es interessant zu sehen, wie sich Organisationen und Familien im Übergang zur funktional differenzierten Gesellschaft als eigene Typen sozialer Systeme ausgebildet haben. Erst mit der Herauslösung aus einer durch Stammes- und Schichtzugehörigkeit geprägten Gesellschaftsformation wurde es möglich, dass Personen verstärkt Mitgliedschaften in unterschiedlichen und voneinander unabhängigen sozialen Systemen ausdifferenzieren konnten. Im Folgenden soll in aller Kürze gezeigt werden, dass die Ausdifferenzierung von Organisationen und Familien als eigene Systemtypen zeitlich weitgehend parallel stattgefunden hat und sich wenigsten teilweise gegenseitig bedingt hat.

Auch wenn eine wichtige Wurzel der Entstehung von Organisationen bereits in jener Zeit liegt, in der sich Religionsgemeinschaften zu religiösen Vereinigungen entwickelten, die ihre Mitglieder aufgrund eigener Entscheidung und - im Gegensatz zu vorher - unabhängig von askriptiven Kriterien wie Familienzugehörigkeit, Schichtzugehörigkeit oder ethnischen Wurzeln rekrutierten (Parsons 1964, S. 347 ff.), kann eine verschiedene gesellschaftliche Felder übergreifende Ausdifferenzierung von Organisationen als einem eigenen Systemtypus erst ab dem 16. und 17. Jahrhundert verortet werden. Erst mit der Ablösung der Politik, des Rechts und der Wirtschaft von der Religion bildeten sich auch in diesen Feldern Organisationen aus, die zunehmend autonom über ihre Mitgliedschaften verfügen konnten (Parsons 1972, S. 88 f.). Besonders mit der Industrialisierung differenzierte sich die Lohnarbeit verstärkt als eine spezifische, von allen anderen Erwartungen befreite, aber in Organisationen gebundene Rolle aus (Parsons 1972, S. 100 ff.; siehe ausführlich auch Türk et al. 2002, S. 148 ff.). Es bildete sich immer mehr ein dominierendes Modell heraus, in welchem Mitgliedschaft in einer Organisation auf einer bewussten Entscheidung sowohl des Mitglieds als auch der Organisation selbst basierte und gleichzeitig Mitglieder - mit Ausnahme totaler Organisationen - nicht mehr mit allen Rollenbezügen in eine Organisation integriert wurden (Lieckweg und Wehrsig 2001, S. 39 ff.). 
Auch die Ausdifferenzierung von Familie als eigenem System fällt in diese Zeit des Übergangs von einer stratifizierten zu einer funktional differenzierten Gesellschaft. In der Tradition von Ernest W. Burgess und Harvey J. Locke lässt sich die Ausdifferenzierung von Familien als ein Übergang der Familie von einem multi- zu einem unifunktionalen System beschreiben. Erzieherische, ökonomische, religiöse und medizinische Funktionen in der modernen Gesellschaft werden, so das Argument von Burgess und Locke, zunehmend auf jeweils einige spezialisierte Institutionen übertragen, während die Familie vorrangig nur noch auf „,gegenseitiger Zuneigung“, „mitfühlendem Verständnis“ und dem „Gemeinschaftsgefühl ihrer Mitglieder“ basiert (Burgess und Locke 1945: vii; meine Übersetzung). Weil politische, religiöse und wirtschaftliche Funktionen zunehmend außerhalb der Familien erfüllt werden, braucht - und dieser Gedanke ist zentral - bei der Eheschließung auf die „Verwandtschaftszusammenhänge“ des jeweiligen Partners keine Rücksicht genommen zu werden (Luhmann 1982, S. 183 f.). ${ }^{5}$ Während also in der stratifizierten Gesellschaft die „Familie noch als den Wechsel der Generation übergreifende Einheit begriffen wurde“, zu der häufig auch noch die Dienerschaft und die Angestellten gezählt wurden, birgt in der funktional differenzierten Gesellschaft jede Eheschließung, ja jede Partnerschaftswahl, die Potenzialität für eine Neugründung einer Familie (Luhmann 1982, S. 163). ${ }^{6}$

\footnotetext{
${ }^{5}$ Erst mit der Geburt von Kindern schließen sich, so Luhmann, die „Herkunftsfamilien der Eltern symbolisch zusammen“, ,um aber mit deren Ehen wieder auf jenes schmale Band einer neuen Verbindung reduziert zu werden“ (Luhmann 1982, S. 183 f.).

${ }^{6}$ Ich halte die Ausdifferenzierung der Familie in der modernen Gesellschaft für einen Musterfall, an dem sich die Stärke dieses hier vorgestellten Ansatzes nachweisen ließe. In der systemtheoretischen Orthodoxie wird Familie lediglich auf der gesellschaftstheoretischen Ebene verortet. Das Problem der „Entfremdung des Individuums“ stelle sich in der modernen Gesellschaft ,auf der ganzen Breite“ und könnte nur über die Ausdifferenzierung eines Funktionssystems „Familie“ gelöst werden (Kieserling 2012). Stattdessen schlage ich abgestimmt mit der allgemeinen Familiensoziologie vor, beim Übergang von der stratifizierten zur funktional differenzierten Gesellschaft eine Ausdifferenzierung von Familien als eigene soziale Systeme zu konstatieren. Die einzelnen Familien erfüllen natürlich eine wichtige gesellschaftliche Funktion bei der Reduzierung der „Entfremdung des Individuums", sie sind aber - ähnlich wie Organisationen oder Gruppen - multireferenziell zu begreifen, weil sie beispielsweise auch Bezüge beispielsweise zu Funktionssystemen der Erziehung (bei der Erziehung der Kinder und - mit Abstrichen - auch der Lebenspartner) oder der Wirtschaft (als Konsum- oder manchmal auch noch Produktionsgemeinschaft) aufweisen. Diese Multireferenzialität von Familien als sozialem System kann durch eine Verortung von Familien als Funktionssystem nicht erkannt werden.
} 
Die noch näher zu überprüfende These ist, dass im Prozess der Ausdifferenzierung die beiden Systemtypen Familien und Organisationen sich als eigene Systemtypen „schärfer gegeneinander profilieren“ (vgl. für die Formulierung Luhmann 1975a, S. 14 für Interaktion, Organisation und Gesellschaft). Luhmanns Argument, dass im Rahmen von Interaktionssystemen ,das konkrete Einfühlungsvermögen“ etwa ,in Form von Intimbeziehungen ins Ungewöhnliche gesteigert werden“ kann, wenn die Interaktion ,nicht mehr von gesellschaftlich durchgehenden Normalitätserwartungen belastet ist" (Luhmann 1975a, S. 16), muss vorrangig auf die Ausdifferenzierung von familienspezifischer Interaktion (und eben nicht der Interaktion per se) zurückgeführt werden. Und auch die „organisatorische Spezifikation des Verhaltens“ kann enorm gesteigert werden, wenn Organisationen nicht mehr nur allgemein von religiösen Aktivitäten, politischen Einstellungen, sportlichen Präferenzen oder freundschaftlichen Loyalitäten des Organisationsmitgliedes absehen können, sondern darüber hinausgehend auch keine Rücksicht auf Loyalitäten der Mitglieder in Familien nehmen müssen.

\section{Folgeprobleme der Differenzierung von Organisation und Familie}

Wenn man von der Ausdifferenzierung von Familien und Organisationen spricht, kann man natürlich darunter - ganz im Sinne der ursprünglichen Intention Niklas Luhmanns - erst einmal die Ausdifferenzierung dieser Systeme gegenüber der Gesellschaft verstehen. Man kann - anders als in stratifizierten Gesellschaften - aus einer gesellschaftlichen Stellung in der Regel keine Ansprüche für eine Position in Organisationen oder Familien ableiten. Auch die Tochter eines einflussreichen Industriefabrikanten muss damit leben, dass sie in der sie beschäftigenden Organisation keine aus ihrer Herkunft ableitbare Ausnahmestellung einnimmt. ${ }^{7}$

Für unsere Zwecke interessiert uns jedoch vorrangig, dass sich Organisationen und Familien in der modernen Gesellschaft auch stärker voneinander

\footnotetext{
${ }^{7}$ Auf die Konsequenz, dass auch die Interaktion als soziales System mit eigenen Logiken sich gegenüber Familien und Organisationen ausdifferenziert, kann ich hier nicht eingehen. Die nicht nur empirisch angeleitete, sondern auch theoretisch eingeordnete soziologische Forschung über Interaktionen in Familien und Interaktionen in Organisationen ist eher unterentwickelt (siehe aber beispielsweise für Interaktion in Familien Keppler 1994, S. 23 ff.; oder für Interaktion in Organisationen Kieserling 1994, S. 168 ff.). Was weitgehend zu fehlen scheint, sind vergleichende Studien über Interaktionen in Familien und Organisationen.
} 
differenzieren. Häufig ohne dass dieses explizit vermittelt wird, lernt man, dass Organisationen und Familien ganz unterschiedlichen Logiken folgen. Während Sokrates für die stratifizierte Gesellschaft noch ganz selbstverständlich davon ausging, dass die Anforderungen an die Führung einer Familie und einer Armee ähnlich seien, weil es darauf ankomme, Untergebene sich „folgsam und gehorsam zu machen“, „die Schlechten zu bestrafen, die Guten zu ehren“ und bei Untergebenen „gute Gesinnung gegen sich zu erwecken“ (Xenophon 1789, S. 111 f.), würde eine solche Position eines Vater oder einer Mutter in der modernen Gesellschaft gelinde gesagt Irritation hervorrufen. ${ }^{8}$ Der Jugendliche, der in einer Organisation wie in einer Familie behandelt werden möchte, wird vermutlich von seiner Umwelt ähnlich skeptisch betrachtet werden wie der Manager, der seine Familie ähnlich führen möchte wie eine Organisation (Dreeben 1968, für den ersten und Loriot (1991) Pappa ante Portas, für den zweiten Fall).

Während in den Selbstbeschreibungen von Familien und Organisationen nicht selten die Vereinbarkeit der Mitgliedschaft im eigenen System mit der Mitgliedschaft in anderen Systemen betont wird - Stichwort ,wir sind ein familienfreundliches Unternehmen“ "-, hebt die Soziologie eher die Spannungen hervor, die sich daraus ergeben, dass Personen den Anforderungen unterschiedlicher sozialer Systeme ausgesetzt sind. Während im Zuge der Ausdifferenzierung von Organisationen wie Armeen, Unternehmen und Schulen in den militärischen, erzieherischen und betriebswirtschaftlichen Reflexionstexten immer wieder darauf verwiesen wurde, dass die Mitgliedschaft in diesen Organisationen selbstverständlich mit einer Mitgliedschaft in einer Familie vereinbar sei, wird gerade in den soziologischen Beschreibungen das Spannungsfeld von Organisation und Familie dargestellt (Treiber und Steinert 1980). Die Spannungen zwischen Schulen und Familien über die Erziehungshoheit für Schüler (Dreeben 1968), die Auseinandersetzungen zwischen Armeen und Familien über die Zugriffsmöglichkeiten auf junge Erwachsene (Shils und Janowitz 1948) und die Debatten in Unternehmen über „Work-Life-Balance“, in denen es in der Regel um Ausbalancierung der Ansprüche von Organisationen und Familien geht (Oechsle 2008), sind nur besonders prominente Beispiele, in denen die Spannungen thematisiert werden, die sich aufgrund der Ausdifferenzierung von Organisation und Familie ergeben haben.

\footnotetext{
${ }^{8}$ Nikomachides äußerte in dem Dialog mit Sokrates auch damals schon Zweifel. Selbstredend liegt der Position Sokrates' ein grundlegend anderes Verständnis von Familie zugrunde. Dementsprechend wird in der deutschen Übersetzung des Xenophon von 1789 für „Oikos“ nicht das Wort „Familie“, sondern das Wort „Hauswesen“ verwendet (Xenophon 1789, S. 112).
} 


\section{Perspektiven - Verschachtelungen, Kombinationen und Übergänge von Familien und Organisationen}

Weil es sich bei Familien und Organisationen um Systeme handelt, die auf der Bestimmung von Mitgliedschaft (oder Nichtmitgliedschaft) von Personen basieren, bietet sich bei der Betrachtung des Zusammenspiels der Systemtypen die in der Soziologie bewährte Darstellung über Rollenkonflikte an. Grundgedanke ist dabei immer, dass es in der modernen Gesellschaft zur Ausdifferenzierung verschiedener sozialer Systeme mit jeweils eigenen Anforderungen kommt. In Ausnahmefällen mag eine einzige Rolle eine Person dominieren - zum Beispiel, wenn eine Person ausschließlich Vater, Bewegungsaktivist, Organisationsmitglied oder guter Kumpel ist - der Regelfall ist jedoch, dass Rollenanforderungen von verschiedenen Systemen an eine Person herangetragen werden. Eine systemtheoretische Perspektive braucht sich jedoch nicht auf die Formulierung von Rollenkonflikten zu beschränken. Sie kann vielmehr systematisch untersuchen, in welcher Form die verschiedenen sozialen Systeme aufeinander Bezug nehmen. Die Möglichkeiten für solche Analysen sollen im Folgenden ausgeführt werden.

Obwohl Organisationen und Familien analytisch erst einmal gleichrangig nebeneinander stehen, können sie auch ineinander verschachtelt werden. Man kann sich Organisationen vorstellen, die Familien beschäftigen, aber auch Familien, die zum Beispiel in Form einer Gaststätte eine Organisation betreiben. Aber aufgrund der unterschiedlichen Möglichkeiten der verschiedenen Systemtypen, ihre Mitgliederzahlen expandieren $\mathrm{zu}$ lassen, sind natürlich einige Verschachtelungen wahrscheinlicher als andere (Geser 1980 zur Soziologie kleiner Systeme). ${ }^{9}$

Weil Kommunikationen mehreren Systemen zugleich angehören können, ist es aber auch vorstellbar, dass Gruppen, Organisationen, Bewegungen und Familien

\footnotetext{
${ }^{9}$ Solche Vorschläge zur Einfügung weiterer Ebenen gewinnen ihre Plausibilität dadurch, dass sie die unterschiedlichen Größenmöglichkeiten der Systemtypen zum Anlass nehmen, Verschachtelungen zu vermuten. Weil Gruppen aufgrund der notwendigen gegenseitigen Personenkenntnis nur schwerlich mehr als dreißig oder vierzig Mitglieder umfassen können, liegt es nahe, sie auf einer „tieferen“ Ebene anzusiedeln als Organisationen, deren Mitgliedsgröße im Prinzip nicht begrenzt ist (natürlich kann die Anzahl von Personen nicht allein schon Auskunft über die Komplexität eines sozialen Systems geben). Auch wenn diese unterschiedlichen Größenmöglichkeiten einige Verschachtelungen (zum Beispiel Gruppen ,in“ Organisationen oder Organisationen „,in“ Bewegungen) wahrscheinlicher machen als andere oder auch gänzlich unwahrscheinlich machen (zum Beispiel Bewegungen ,in“ Familien), so braucht man sich nicht unbedingt nur einen Typus von Verschachtelung vorzustellen.
} 
nicht ineinander verschachtelt werden, sondern ohne Primat eines sozialen Systems ineinander verwoben sind. Man denke nur an die Gründung eines Unternehmens durch eine Familie. Solange keine zusätzlichen Angestellten eingestellt werden, ist es wahrscheinlich, dass weder die Familien- noch die Organisationslogik dominiert. ${ }^{10}$

Man kann sich verschiedene Formen von Übergängen von einem Systemtypus zu einem anderen vorstellen. Man denke nur an Familie, aus der gemeinsame wirtschaftliche Aktivitäten in Form eines Familienunternehmens entstanden sind, und von dem nach der Trennung der Eltern nur noch eine Organisation übrig bleibt. Oder man denke an eine Kleinstorganisation aus zwei Personen, in der sich die beiden ineinander verlieben, und als eine um Kinder erweiterte Familie auch dann zusammenbleiben, wenn die Kleinstorganisation sich auflöst.

\section{Literatur}

Burgess, E. W. \& Locke, H. J. (1945). The Family. From Institution to Companionship. New York: American Book Company.

Caplow, T. (1964). Principles of Organization. New York: Harcourt.

Dreeben, R. (1968). On What is Learned at School. Reading: Addison-Wesley.

Fuchs, P. (1997). Adressabilität als Grundbegriff der soziologischen Systemtheorie. Soziale Systeme, 3, (S. 57-80).

Geser, H. (1980). Kleine Sozialsysteme: Strukturmerkmale und Leistungskapazitäten. Versuch einer theoretischen Integration. Kölner Zeitschrift für Soziologie und Sozialpsychologie, 32, (S. 205-239).

\footnotetext{
${ }^{10}$ Gerade der Fall des Familienunternehmens eignet sich um die Vorteile des vorgestellten Vorhabens zu behandeln (Schlippe et al. 2017; Simon 2012). Wenn man das Thema Familienunternehmen - wie von Kieserling (2012) vorgeschlagen - als eine Geschichte der funktionalen Ausdifferenzierung von Wirtschaft und Familien auf Gesellschaftsebene beschreibt, dann kann man lediglich konstatieren, dass es mit der Ausbildung von Aktiengesellschaften $\mathrm{zu}$ einer „rationaleren Verwaltung wirtschaftlichen Eigentums kommt“, weil diese von ,sentimentalen Rücksichten auf die Familientradition befreit“. Familienunternehmen erscheinen aus Sicht nur noch als Überbleibsel vormoderner Gesellschaften. Dieser modernisierungstheoretische Zugang tendiert dazu, die Prominenz von Familienunternehmen im Wirtschaftssystem aufgrund einer theoretischen Setzung - Familien sind ein Funktionssystem und differenzieren sich gegen andere Funktionssysteme wie die Wirtschaft aus - zu ignorieren. Erst wenn man die einzelne Familie als Sozialsystem mit eigenen Logiken begreift, bekommt man die für die Betrachtung von Familienunternehmen relevanten Aspekte in den Fokus.
} 
Gilgenmann, K. (1994). Romantische Liebe und Liebe zum Kind. Zur Differenzierung der Codierung von Partnerschaft und Elternschaft. In A. Herlth et al. (Hrsg.): Abschied von der Normalfamilie? Partnerschaft contra Elternschaft, (S. 64-82). Berlin: Springer.

Keppler, A. (1994). Tischgespräche. Über Formen kommunikativer Vergemeinschaftung am Beispiel der Konversation in Familien. Frankfurt a. M.: Suhrkamp.

Kieserling, A. (1994). Interaktion in Organisationen. In K. Dammann (Hrsg.), Die Verwaltung des politischen Systems (S. 168-182). Opladen: Westdeutscher Verlag.

Kieserling, A. (2012). Notizen zu einem Vorschlag von Stefan Kühl, Luhmanns Ebenenunterscheidung betreffend. Bielefeld: unveröff. Ms.

Lieckweg, T. \& Wehrsig, C. (2001). Zur komplementären Ausdifferenzierung von Organisationen und Funktionssystemen. Perspektiven einer Gesellschaftstheorie der Organisation. In V. Tacke (Hrsg.), Organisation und gesellschaftliche Differenzierung (S. 39-60). Opladen: Westdeutscher Verlag.

Pappa ante Portas. (Deutschland 1991). Regie: Loriot. DVD/Blu-ray Warner Home Video 2001.

Luhmann, N. (1964). Funktionen und Folgen formaler Organisation. Berlin: Duncker \& Humblot.

Luhmann, N. (1973). Zweckbegriff und Systemrationalität. Über die Funktion von Zwecken in sozialen Systemen. Frankfurt a. M.: Suhrkamp.

Luhmann, N. (1975a). Interaktion, Organisation, Gesellschaft. In N. Luhmann (Hrsg.), Soziologische Aufklärung 2. Aufsätze zur Theorie der Gesellschaft (S. 9-20). Opladen: Westdeutscher Verlag.

Luhmann, N. (1975c). Macht. Stuttgart: Enke.

Luhmann, N. (1982). Liebe als Passion. Zur Codierung von Intimität. Frankfurt a. M.: Suhrkamp.

Luhmann, N. (1991). Soziologie des Risikos. Berlin; New York: Walter de Gruyter.

Luhmann, N. (2002). Das Erziehungssystem der Gesellschaft. Frankfurt a. M.: Suhrkamp.

Luhmann, N. (2005a). Sozialsystem Familie. In N. Luhmann (Hrsg.), Soziologische Aufklärung 5. (S. 189-209). Opladen: Westdeutscher Verlag.

Luhmann, N. (2005b). Glück und Unglück der Kommunikation in Familien. In N. Luhmann (Hrsg.), Soziologische Aufklärung 5. (S. 210-219). Wiesbaden: VS Verlag.

Mahlert, B. (2011). Mitgliedschaftsbasierte Sozialsysteme in der Weltgesellschaft. Bielefeld.

Martens, W. (1997). Organisation als gesellschaftliche Teilsysteme. In G. Ortmann, J. Sydow \& K. Türk (Hrsg.), Theorien der Gesellschaft. Die Rückkehr der Gesellschaft (S. 263-311). Opladen: Westdeutscher Verlag.

Mayntz, R. \& Ziegler, R. (1977). Soziologie der Organisation. In R. König (Hrsg.), Handbuch der empirischen Sozialforschung. Band 9 (S. 1-141). Stuttgart: Enke.

Oechsle, M. (2008). Work-Life-Balance: Diskurse, Problemlagen, Forschungsperspektiven. In R. Becker \& B. Kortendiek (Hrsg.), Handbuch Frauen- und Geschlechterforschung. Theorie, Methoden, Empirie (S. 227-236). Wiesbaden: VS Verlag.

Parsons, T. (1964). Evolutionary Universal in Society. In American Sociological Review, 29, (S. 339-357).

Parsons, T. (1972). Das System moderner Gesellschaften. Weinheim; München: Juventa.

Schlippe, A. v., Groth, T. \& Rüsen, T. A. (2017): Die beiden Seiten der Unternehmerfamilie. Familienstrategie über Generationen: Auf dem Weg zu einer Theorie der Unternehmerfamilie. Göttingen: Vandenhoeck \& Ruprecht. 
Shils, E. A. \& Janowitz, M. (1948). Cohesion and Disintegration in the Wehrmacht in World War II. The Public Opinion Quarterly, Sommer 1948, (S. 280-315).

Simon, F. B. (2012). Einführung in die Theorie des Familienunternehmens. Heidelberg: Carl Auer.

Stichweh, R. (2000). Adresse und Lokalisierung in einem globalen Kommunikationssystem. In R. Stichweh (Hrsg.), Weltgesellschaft. Soziologische Analysen (S. 220-231). Frankfurt a. M.: Suhrkamp,

Tacke, V. (2000). Netzwerk und Adresse. Soziale Systeme, 6, (S. 291-320).

Treiber, H. \& Steinert, H. (1980). Die Fabrikation des zuverlässigen Menschen. Über die „,Wahlverwandtschaft“ von Kloster- und Fabrikdisziplin. München: Heinz Moos Verlag.

Türk, K., Lemke, T. \& Bruch, M. (2002). Organisation in der modernen Gesellschaft. Opladen: Westdeutscher Verlag.

Tyrell, H. (1983b). Familie als Gruppe. Kölner Zeitschrift für Soziologie und Sozialpsychologie, Sonderheft 25, (S. 362-390). Opladen.

Tyrell, H. (2008). Zweierlei Differenzierung: Funktionale und Ebenendifferenzierung im Frühwerk Niklas Luhmanns. In B. Heintz (Hrsg.), Soziale und gesellschaftliche Differenzierung. Aufsätze zur soziologischen Theorie (S. 75-105). Wiesbaden: VS Verlag.

Tyrell, H. \& Herlth, A. (1994). Partnerschaft versus Elternschaft. In A. Herlth et al. (Hrsg.), Abschied von der Normalfamilie? Partnerschaft kontra Elternschaft (S. 1-15). Berlin u. a.: Springer Verlag.

Xenophon (1789). Sokrates. Leipzig: Schwickerscher Verlag. 